\title{
Effects of Cannabis on Cardiovascular System: The Good, the Bad, and the Many Unknowns
}

\author{
Ali E. Dabiria, Ghassan S. Kassab ${ }^{a}$

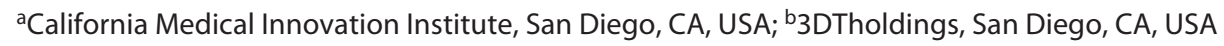

\section{Keywords}

Smoking · Marijuana · Synthetic cannabinoids · Illicit substance

\begin{abstract}
Cannabis is currently the most consumed illicit substance in the world, and gradual legalization in the USA makes it important to understand the health consequences of the use of this substance. With growing body of evidence that some cannabis ingredients may be beneficial in various aspects of hemostasis, additional research is clearly needed in various clinical areas. In addition to understanding the efficacy, research efforts should also include studies that address any harmful effects of the compounds or administration methods that may result in adverse effects. This review is focused on the cardiometabolic effects of cannabis use. Cardiometabolic diseases are among the leading causes of death in the USA and around the world. The purpose of this review was to provide an overview of the known medicinal benefits of selected cannabis cannabinoids and the known side effects or contraindications. More importantly, we have proposed new questions and signposts in cannabis research to uncover additional medicinal benefits and identify the health hazards with focus on cardiovascular disease.
\end{abstract}

(c) 2021 The Author(s).

Published by S. Karger AG, Basel

karger@karger.com

www.karger.com $/ \mathrm{mca}$

Karger ${ }^{\prime \prime}$ -

BOPEN ACCESS
(C) 2021 The Author(s)

Published by S. Karger AG, Basel

This is an Open Access article licensed under the Creative Commons Attribution-NonCommercial-4.0 International License (CC BY-NC) (http://www.karger.com/Services/OpenAccessLicense), applicable to the online version of the article only. Usage and distribution for commercial purposes requires written permission.

\section{Introduction}

The legalization of medical and recreational cannabis in the world has coincided with increases in various methods of cannabis consumption which makes it important to understand the health consequences. The global legal cannabis market size is expected to reach USD 84B by the end of 2028, according to a report by Grand View Research [1]. The cannabis market is expected to expand at a compound annual growth rate of $14 \%$ from 2021 to 2028 according to the report. Gallup poll indicates that Americans' support for legalizing marijuana has been around $66 \%$ in 2018 , after rising $30 \%$ points between 2005 and 2018 [2]. In a 2019 Gallup poll, 13\% of US adults reported smoking cannabis, a percentage which was almost double the percentage reported 3 years earlier [3]. About $43 \%$ of adults in the USA reported having tried cannabis in $2019,44 \%$ in $2018,38 \%$ in 2013 , and $4 \%$ in 1969 [3].

Cannabis is the most complex plant known to humans with many active ingredients, most of which have largely unknown health effects. In this review, we have provided an overview of the known medicinal benefits of selected cannabis cannabinoids and the known side effects or contraindications. More importantly, we have proposed new questions and signposts in cannabis research to uncover additional medicinal benefits and identify the health hazards with focus on cardiovascular disease (CVD). 


\section{National Academies of Science Report}

A committee on the health effects of cannabis consumption was formed at the National Academies of Sciences, Engineering and Medicine, to extensively review the scientific literature and identify the research gap recommendations. The committee was formed by 16 experts in the areas of cannabis addiction, oncology, cardiology, neurodevelopment, respiratory disease, pediatric and adolescent health, immunology, toxicology, preclinical research, epidemiology, systematic review, and public health. Given the large amount of scientific literature on cannabis, the committee developed an approach that resulted in giving primacy to published systematic reviews (since 2011) and high-quality primary research for 11 groups of health endpoints, including cardiovascular conditions. The report was published in January 2017 [4].

The objective of the review of cannabis and cardiometabolic conditions was to assess the independent relationship of cannabis use with these conditions in studies where the relationship has been quantified. The reason for examining cannabis use in relation to cardiometabolic conditions is that these conditions are among the leading causes of mortality; are highly widespread in the USA; account for high level of medical care and cost; and are mainly caused, by changing lifestyle, including poor diet, lack of physical activity, and cigarette smoking. It is anticipated that legalization expansion of cannabis will cause the rates of consumption to increase [4].

The committee summarized the cardiometabolic literature published since 1999 [4]. The committee found little evidence of a relationship between acute cannabis consumption - but not chronic cannabis consumption and acute myocardial infarction (MI) risk. The committee also established that there is limited evidence of a relationship between cannabis consumption and an increased risk of ischemic stroke or subarachnoid hemorrhage. The committee also found no data on sudden mortality and arrhythmias such as atrial fibrillation to quantify the relationship with cannabis consumption. The limitations of the reviewed studies included lack of data on different methods of cannabis consumption (e.g., smoke and edible ), inadequate dose information, little information on potential additives or contaminants, and lack of adequate data on total lifetime duration/dose of cannabis consumption [4].

The committee identified major gaps and opportunities that relate to the insufficient amount of longitudinal data for all the cardiometabolic disorders and lack of data on the impact of cannabis use on risk in the older age groups where most of the CVD occur (e.g., acute MI [AMI] and stroke). The committee suggested the following to address the current gaps: (1) establish population cohort(s) in which cannabis consumption is regularly evaluated with standardized questionnaires accounting for the type of cannabis preparation, cannabis strength, the amount of consumption, frequency and duration of consumption, and CVD risk data and collect medical and toxicology data on incident of CVD events; (2) the cohort(s) need to be large enough such that the relationship of cannabis consumption with CVD events can be statistically significant; and (3) categorize the events in electronic health records [4].

\section{Methods to Consume Cannabis}

Cannabis is consumed in several ways which involve heating decarboxylate tetrahydrocannabinol acid in the plant into tetrahydrocannabinol (THC) [5]. THC is oil and slightly water soluble. The following are some methods of consumption: (1) smoking involves inhaling vaporized cannabinoids from small pipes, bongs (portable versions of hookahs with a water chamber), paperwrapped joints or tobacco-leaf-wrapped blunts; (2) vaporizer, where any form of cannabis can be heated to $165-190^{\circ} \mathrm{C}$, which causes the active ingredients to evaporate; (3) cannabis tea which contains small amount of THC. It is prepared by adding a saturated fat to hot water (e.g., cream or any milk except skim) with a small amount of cannabis; (4) edibles, containing cannabis which is mixed with one of a variety of foods; (5) capsules where THC and other dietary supplement products are mixed.

Legalization of medical and recreational cannabis has incentivized consumers to develop novel forms of cannabis consumption. Meacham et al. [6] researched these emerging and traditional forms of consumption in an online social media discussion forum. They reported smoking was the most common form of cannabis consumption (about 18\% of 2.26 million posts). They found that, from 2010 to 2016, additional post volume per year increased for posts noting dabbing $(3.63 / 1,000)$, butane hash oil $(3.16 / 1,000)$, and edible $(2.84 / 1,000)$.

\section{Mechanism of Action for Cannabis}

The endocannabinoid system consists of the endogenous cannabinoids, cannabinoid receptors, and the enzymes that synthesize and degrade cannabinoids. Many
Dabiri/Kassab 
of the effects of cannabinoids are mediated by $2 \mathrm{G}$ protein-coupled receptors, $\mathrm{CB}_{1}$ and $\mathrm{CB}_{2}$, although additional receptors may be involved [7]. $\mathrm{CB}_{1}$ receptors are present in high levels in several brain regions and in lower amounts in a more widespread distribution. These receptors mediate many of the psychoactive effects of cannabinoids. $\mathrm{CB}_{2}$ receptors have a more restricted distribution, being found in several immune cells and in a few neurons. Both $\mathrm{CB}_{1}$ and $\mathrm{CB}_{2}$ coupled primarily to $\mathrm{G}$ proteins and are subject to the same pharmacological influences as other G protein-coupled receptors. Thus, partial agonism, inverse agonism, and functional selectivity all play important roles in determining the cellular response to specific cannabinoid receptor ligands [7]. These receptors are crucial to utilizing the active components in cannabis that influence homeostasis. The identification of cannabinoid receptors stemmed from the desire to understand the psychoactive effects of THC, the principal psychoactive component of cannabis. THC is the main activator of $\mathrm{CB}_{1}$ through allosteric modulators, which can potentially allow the therapeutic effects of THC without the intoxicating effects. $\mathrm{CNR}_{1}$ gene produces the $\mathrm{CB}_{1}$ protein. Since each individual carries a different version of the $\mathrm{CNR}_{1}$ gene, many people have a different experience with the use of compounds, like THC and cannabidiol (CBD).

Various cannabinoids have different effects on the receptors, functioning as agonists, antagonists, or partial antagonists, as well as affecting the vanilloid receptor. Many known effects of cannabis consumption can be explained based on this mechanism of action in various conditions including multiple sclerosis, Parkinson's disease, glaucoma, nausea, vomiting, and rheumatoid arthritis [7]. There are many other receptors working with cannabinoids.

\section{Rationale for Studies of Cardiovascular Disease}

Although cannabis use may impact numerous organ systems (brain and pulmonary, skeletal), we focus on the cardiovascular (CV) system. An estimated $86 \mathrm{M}$ American adults have at least one CVD, such as heart disease, stroke, heart failure, or hypertension [8]. Heart disease is the leading cause of mortality in the USA, accounting for $>600 \mathrm{~K}$ deaths per year in 2014 , or $23 \%$ of all death in the USA [9]. Within subcategories of heart disease, coronary heart disease (CHD) is by far the largest [9]. CHD is a disease where plaque builds up inside the blood vessels (i.e., the coronary arteries) supplying the heart. Over the

Effects of Cannabis on the Cardiovascular System course of years or decades, the plaque can harden or rupture, resulting in an inadequate supply of blood to the heart which may result in death of the heart muscle, like MI. Each year, an estimated 550K Americans have an incident (i.e., first time) heart attack (AMI) and about $200 \mathrm{~K}$ have a recurrent attack [8]. Of those who have a heart attack each year, about $116 \mathrm{~K}$ die as a result [8]. Cardiometabolic disorders result in a substantial economic burden on the USA. The annual total cost of CVD, including heart disease, stroke, hypertensive disease, and other circulatory conditions in the USA was estimated at USD $351 \mathrm{~B}$ in 2014-2015, with USD 214B in direct cost, including $46 \%$ for inpatient care [10]. Given the epidemics of CVD, the effects of cannabis on cardiometabolic disorders are obviously important.

\section{Benefits Associated with Cannabis}

The US Food and Drug Administration (FDA) does not regulate $\mathrm{CBD}$ for most medical conditions. Therefore, dosages recommended are open to question and it is important to be on the safe side. Under the Controlled Substances Act (CSA), CBD is currently a schedule I substance because it is a natural chemical component of the cannabis plant. The FDA has approved 3 drugs based on cannabinoids. Dronabinol, the generic name for synthetic THC, is marketed under the trade name of Marinol ${ }^{\circledR}$ and is clinically indicated to counteract the nausea and vomiting associated with chemotherapy and to stimulate appetite in AIDS patients affected by wasting syndrome. A synthetic analog of THC and nabilone (Cesamet ${ }^{\circledR}$ ), is prescribed for similar indications. Both dronabinol and nabilone are given orally and have a slow onset of action. In July 2016, the FDA-approved Syndros ${ }^{\circledR}$, a liquid formulation of dronabinol, for the treatment of patients experiencing chemotherapy-induced nausea and vomiting who have not responded to conventional antiemetic therapies. The agent is also indicated for treating anorexia associated with weight loss in patients with AIDS. Two additional cannabinoid-based medications have been examined by the FDA. Nabiximols (Sativex ${ }^{\circledR}$ ) is an ethanol cannabis extract composed of THC and CBD as active components. Nabiximols are administered as an oromucosal spray and indicated in the symptomatic relief of multiple sclerosis and as an adjunctive analgesic treatment in cancer patients [11]. As of 2018, nabiximols have been launched in several countries, including the USA, Canada, Germany, Italy, Spain, the UK. In response to the urgent need expressed by parents of children with intrac- 
table epilepsy, FDA-approved Epidiolex (brand name) CBD oral solution for the treatment of seizures associated with 2 rare and severe forms of epilepsy, Lennox-Gastaut syndrome and Dravet syndrome, in patients 2 years of age and older [12]. This is the first FDA-approved drug that contains a purified drug substance derived from cannabis, CBD. It is also the first FDA approval of a drug for the treatment of patients with Dravet syndrome. The patient information leaflet for Epidiolex cautions that there is a risk of liver damage, lethargy, and possibly depression and thoughts of suicide, but these are also true of other treatments for epilepsy. Stanley et al. [13] reviewed the effects of CBD in the cardiovascular system.

In isolated arteries, direct application of $\mathrm{CBD}$ causes both acute and time-dependent vasorelaxation of preconstricted arteries and enhances endothelium-dependent vasorelaxation in models of endothelial dysfunction. In vivo, CBD treatment does not appear to have any effect on resting blood pressure or heart rate but does reduce the cardiovascular response to various types of stress. In vivo, $\mathrm{CBD}$ treatment has a protective role in reducing the effects of cardiac ischemia and reperfusion, or in reducing cardiac dysfunction associated with diabetes. Similarly, $\mathrm{CBD}$ has a protective role in reducing the ischemic damage in models of stroke, partly due to maintaining cerebral blood flow. In models of altered vascular permeability, CBD reduces the hyperpermeability of the blood retinal barrier in diabetes and blood-brain barrier hyperpermeability after lipopolysaccharide injection. Similarly, CBD ameliorates the negative effects of a high glucose environment on cell adhesion molecules and barrier function. Together, these data suggest that the cardiovascular system is indeed a valid therapeutic target for $\mathrm{CBD}$. The target sites of action for CBD, however, remain to be established for most of these responses. Whether these responses to CBD will translate into the human cardiovascular system remain to be seen. For example, Jadoon et al. [14] investigated if CBD reduces blood pressure in 9 healthy male volunteers given $600 \mathrm{mg}$ of CBD or placebo in a randomized, placebo-controlled, doubleblind, crossover study. Cardiovascular parameters were monitored using a finometer and laser Doppler. They concluded that acute administration of CBD reduces resting blood pressure. Although the results were promising, further research is required to establish whether CBD has a role in the treatment of cardiovascular disorders.

There are other promising applications for CBD-like smoking cessation [15], drug withdrawal treatment [16], treating seizures and epilepsy [17], anxiety treatment [18], reducing some of the effects of Alzheimer's [19], and antipsychotic effects on patients with schizophrenia [20]. Future applications include combating type I diabetes [21] and cancer treatment $[22,23]$. Anecdotal evidence suggests that this is a promising and versatile treatment, although more research is needed. Applications outside of CV area will not be discussed here.

\section{Problems Associated with Cannabis and/or Synthetic Cannabinoids}

The large market of cannabis has given rise to numerous potentially hazardous natural contaminants or artificial adulterants being reported in crude cannabis and preparations. Most frequent natural contaminants consist of microbial contamination (e.g., fungi and bacteria), pesticides, and heavy metals [24]. These contaminants are usually introduced during cultivation and storage [24]. Growth enhancers and pest control chemicals are the most common risks to both producer and consumer [24]. Cannabis can also be contaminated for profit purposes which entails adding substances (e.g., tiny glass beads and lead) to increase the weight of the cannabis product $[25,26]$. In some case, psychotropic substances (e.g., tobacco and calamus) and cholinergic compounds are added to either enhance the efficacy of low-quality cannabis or to alleviate its side effects [27]. Additionally, some extraction methods can result in pesticide and solvent contamination [28]. Furthermore, certain methods used for inhalation (e.g., vaporizing and "dabbing") can cause the formation of potentially harmful byproducts of combustion (e.g., formaldehyde and acrylamide), of which the long-term effects of inhalation are unknown [28]. Hence, rigorous and standardized testing of cannabis content is critical to mitigate potential preventable toxicity [28].

In contrast to THC, which is a partial agonist of the $\mathrm{CB}_{1}$ receptor, many of the synthetic cannabinoids bind to $\mathrm{CB}_{1}$ receptors with high affinity and efficacy, which may also be associated with higher potential of toxicity [29]. These agents have become popular as black-market alternatives to cannabis because they were previously legal in many locals and have been undetectable in urine drug screens where THC metabolites may remain for weeks [30]. Routine users may experience withdrawal and symptoms of dependence [31]. According to the National Institute on Drug Abuse [32], people using these various blends have been admitted to Poison Control Centers reporting "rapid heart rate, vomiting, agitation, confusion, and hallucinations." Synthetic cannabinoids can DOI: 10.1159/000519775
Dabiri/Kassab 
also raise blood pressure and cause a reduced blood supply to the heart (myocardial ischemia), and in some cases, they have been associated with heart attacks. Routine users may experience withdrawal and symptoms of dependence [32].

Use of synthetic cannabinoids is gradually increasing in the world due to ease of accessibility, relatively low cost, and inability to show usage by routine toxicological screening methods [33]. The CV side effect related to use of synthetic cannabinoids "bonzai" has been reported for 2 cases [33]. With presentation of these cases, the researchers [33] aimed to emphasize that synthetic cannabinoids "bonzai" addicts the number of whom increases gradually may present with different $\mathrm{CV}$ system findings. Clearly more studies related with the effects of use of synthetic cannabinoids "bonzai" on the CV system are needed. An in-depth review [34] has examined the CV effects of synthetic cannabinoids to conclude that further research is needed to document the regulatory measures that are needed to curb the spread of synthetic cannabinoids. It is essential to keep the risk/benefit ratio of the medicinal use of cannabis as low as possible.

\section{Unknowns Associated with Cannabis Consumption}

It is imperative that cannabis legalization which will likely increase cannabis use, does not cause significant adverse effect, like tobacco smoking. Public policy needs to be formed around a rigorous study to investigate any adverse health effect of cannabis smoking. Discussion about whether smoke-free legislations should also include cannabis is becoming increasingly widespread while the industry is growing.

There are several mechanisms by which cannabis consumption can contribute to the development of chronic CVD or acutely trigger CV events [35]: (1) pro-arrhythmic effects of catecholamines, the production of which is stimulated by THC; (2) increase of heart rate that can result in cardiac ischemia in susceptible individuals; (3) analgesic properties of THC that may lead to treatment delay for chest pain; (4) increased blood carboxy-hemoglobin levels resulting in decreased oxygen supply for the heart, which results in increase of heart rate; and (5) lability in blood pressure that can increase risk of transient ischemic attack) or stroke in individuals with CVD [35]. In addition, cannabis smoking produces oxidant gases that contribute to cellular stress and may contribute to $\mathrm{CV}$ risk by activating platelets, promoting formation of oxidized low-density lipoprotein, and inducing an in-

Effects of Cannabis on the Cardiovascular System flammatory response [36]. Cannabis consumption may be related to the incidence of CVD through associations with traditional CV risk factors, including blood pressure level and lipids. Data regarding the association of cannabis consumption with chronic levels of these risk factors, however, are not available. Consideration of the cannabis-tobacco consumption relationship is critical in any analysis of the association of cannabis use to tobacco-related diseases, including many of the CVD [35].

Cannabis is also related to less well-known correlates including impaired pulmonary function leukocyte count and hostility [37]. Lung function abnormalities, including decrements in several lung function parameters, can occur with chronic consumption of cannabis [37, 38]. Pulmonary function is inversely related to the incidence of CHD and congestive heart failure, although it is not known whether this relationship is causal in nature [39]. The circulating leukocyte count is related to the incidence of MI [40], while hostility is related to CHD mortality and coronary artery calcification, an early indicator of coronary atherosclerosis $[41,42]$. The leukocyte count was related to cannabis consumption in the CARDIA study, conducted in a biracial study population of $>5,000$ young adults [43]. High hostility level (as determined by the Cook-Medley Hostility Scale, a self-administered questionnaire) was strongly related to cannabis consumption in the CARDIA study [43].

One of the very first studies to investigate the effect of smoking cannabis on MI was conducted in 2000 [44]. Smoking cannabis is known to have hemodynamic consequences, including a dose-dependent increase in the heart rate, supine hypertension, and postural hypotension. Whether it can trigger the onset of MI was unknown. In this investigation, 3,882 patients ( 1,258 women) were interviewed with AMI on an average of 4 days after infarction onset. They used the case-crossover study design to compare the reported consumption of cannabis in the hour preceding symptoms of MI onset to its expected frequency using self-matched control data. Of the 3,882 patients, 124 (3.2\%) reported smoking cannabis in the prior year, 37 within $24 \mathrm{~h}$ and 9 within $1 \mathrm{~h}$ of MI symptoms. The risk of MI onset was elevated 4.8 times over nonusers ( $95 \%$ confidence interval) in $1 \mathrm{~h}$ after cannabis consumption. The elevated risk rapidly decreased thereafter. They concluded that smoking cannabis was a rare trigger of AMI and may pose a health risk to patients with established coronary artery disease and perhaps to individuals with multiple coronary risk factors. The study had several limitations including some misclassification of exposure likely to have occurred since the data were based on 
patient self-report. For example, patients may be reluctant to report that they had consumed cannabis before their MI. There was also a possibility of bias caused by differential survival of cases that had their MI triggered by different mechanisms. For example, if patients whose infarctions were triggered by cannabis were more likely to die than those whose infarctions were unrelated to cannabis, then the apparent relative risk may be underestimated.

Gleerup et al. [45] investigated the risk factor of the tobacco-smoking habits in younger patients with ST-segment elevation myocardial infarction (STEMI). The study demonstrated that 3 out of 4 younger patients with STEMI were current smokers and only $16 \%$ of the patients had never smoked. Smoking was the most prevalent modifiable risk factor in younger patients with STEMI, and notably was most frequent among women. The study indicated that despite efforts to reduce the prevalence of smoking, it remains the most dominating risk factor in these patients. Furthermore, the youngest patients may benefit the most from avoiding STEMI, which has major consequences at both patient and society level. Cannabis is often smoked in combination with tobacco which makes it difficult to imply the association of only cannabis smoking with MI and ischemic stroke. This argument is also relevant for cannabis second-hand smoking. There is clearly a gap in knowledge to distinguish between effects related to tobacco smoking and those related to cannabis intake.

An additional study suggested a link between cannabis consumption and the development of $\mathrm{MI}$ in patients with coronary artery disease [46-48]. Mukamal et al. [49] studied cannabis consumption and mortality following AMI. The preliminary results indicated possible hazards of cannabis for patients who survive AMI. They also indicated that although cannabis use had not been related to mortality in other populations, it may pose particular risk for susceptible individuals with coronary heart disease. Frost et al. [50] studied cannabis consumption and long-term mortality among survivors of AMI. They concluded that habitual cannabis consumption among patients presenting with AMI was related to an apparent increased mortality rate over the following 18 years that did not reach statistical significance. They recommended that larger investigations with repeated measures of cannabis consumption are needed to definitively instigate whether there are adverse CV outcomes of smoking cannabis among patients with coronary heart disease.

A study was conducted in France from 2006 to 2010 to investigate the $\mathrm{CV}$ complications related to cannabis and their extreme seriousness in 1979 cannabis-related cases [51]. The percentage of cannabis-related CV complications increased from $1.1 \%$ in 2006 to $3.6 \%$ in 2010 of all cannabis-related reports with a death rate of $25.6 \%$. They were all serious and included cardiac and extracardiac complications, mainly acute coronary syndromes and peripheral arteriopathies. The results indicated that cannabis as a possible risk factor for CVD in young adults was in agreement with previous results. The authors concluded that clinicians should be aware that cannabis consumption in young people can be a potential triggering factor for $\mathrm{CV}$ complications and the data concerning their danger must be widely distributed.

In another study [52], the investigators showed that cannabis cigarettes cause increases in heart rate, supine systolic and diastolic blood pressures compared with placebo. These actions increase myocardial oxygen demand to a degree that they can decrease the time to exerciseinduced angina in patients with a history of stable angina. In addition, cannabis has been related to triggering MIs in young male patients. Smoking cannabis has been shown to increase the risk of MI onset by a factor of 4.8 for $60 \mathrm{~min}$ after cannabis use, and to increase the annual risk of $\mathrm{MI}$ in the daily cannabis user from 1.5 to $3 \%$ per year. Human and animal models suggest that this effect may be due to coronary arterial vasospasm. Longitudinal studies, however, have indicated that cannabis consumption may not have a significant effect on long-term mortality. Researchers concluded that further work is required to definitively establish the impact of cannabis on $\mathrm{CVD}$, and it is reasonable to recommend against recreational cannabis consumption, especially in individuals with a history of coronary artery disorders.

Marchetti et al. [53] attempted to find evidence of MI due to coronary thrombosis in an autopsy of an occasional cannabis smoker. They performed a narrative review of the literature to establish when postmortem toxicological tests may support a temporal association between cannabis smoking and CVD. Toxicological examination showed the presence of D9-tetrahydrocannabinol, its main metabolite, and cannabinol in blood and urine. Semi-quantitative analysis revealed that D9-THC was taken within $2 \mathrm{~h}$ of the onset of CV symptoms, according to circumstantial data. For any conclusion to be made about the specific CV-triggering effect of D9-THC intake, however, they realized that cannabinoid analysis in blood samples must be considered an essential requirement to estimate the time of last intake to avoid incomplete documentation. The investigators highlighted a relationship between cannabis consumption and negative $\mathrm{CV}$ events; although
Dabiri/Kassab 
few authors did support their findings with toxicological results and therefore concluded further research is needed [53].

Rumalla et al. [54] sought a correlation between cannabis consumption and hospitalization for acute ischemic stroke (AIS) using data from the largest inpatient database in the USA. Cannabis consumption $(N=$ $2,496,165)$ and noncannabis consumption ( $N=$ $116,163,453$ ) cohorts were identified (age 15-54) in the Nationwide Inpatient Sample (NIS) from 2004 to 2011. Incidence of AIS was significantly greater in the cannabis cohort than the noncannabis cohort in all age groups. The incidence of AIS hospitalization in cannabis consumers and noncannabis consumers was established. They utilized multivariable logistic regression analyses to study the independent relationship between cannabis consumption and AIS. Overall, the incidence of AIS was significantly greater among cannabis consumers than nonconsumers and had the greatest difference in the 25-34 age group. Cannabis consumption was more prevalent among younger patients, males, African-Americans, and Medicaid enrollees. Cannabis consumers were more likely to use other illicit substances but had less overall medical comorbidity. They concluded that recreational cannabis consumption is independently related to $17 \%$ increased likelihood of AIS hospitalization among younger adults.

There is lack of concrete evidence for cannabis secondhand smoking (CSHS) causing acute CV harm. There have been some investigations in rats [55] whether exposure to cannabis CSHS can cause acute vascular endothelial dysfunction. The results indicated that $1 \mathrm{~min}$ of exposure to CSHS impaired flow-mediated dilation to the same extent as impairment from equal exposure of tobacco CSHS. There were several limitations to this study. For example, ambient concentration of cannabis CSHS have not been systematically measured in real conditions, opposite to tobacco CSHS. Another limitation is that in a rodent model, parameters such as the exposure times and durations of impairment may not completely translate to exposure in humans. It should be noticed that rats and humans show similar responses comparable to tobacco smoke exposure conditions. The report [55] concludes that a prospective-controlled rodent experiment can fill a gap in understanding of the consequences of cannabis CSHS exposure that can be extrapolated to humans since understanding of human CV consequences of cannabis use has been limited to retrospective studies. Additionally, other forms of noncombusted, inhaled cannabis products also can be investigated (vaporized dried can-

Effects of Cannabis on the Cardiovascular System nabis and vaporized cannabis extracts), to evaluate any potential CSHS exposure risks of alternate forms of cannabis and exposure to tobacco will be used as a control for comparison. In summary, the findings indicate that in rats, flow-mediated dilation is damaged by some constituents of smoke not related to cannabis or tobacco. It could be either the products of combustion or other plant chemicals that exist after combustion. Therefore, the mechanism by which tobacco and cannabis smoke damage endothelial function is not fully understood.

Finally, investigators studied the National Inpatient Sample, the largest publicly accessible database in the USA, to survey the number of hospital admissions in patients with a history of heart attack, percutaneous coronary intervention and/or coronary artery bypass graft, and self-identified cannabis consumers and nonconsumers from 2007 to 2014 [56]. The database includes about $8 \mathrm{M}$ hospital stays each year and does not include information about forms of cannabis usage. Many states in the USA legalized medical/recreational cannabis use during the study's period. The findings were as follows: (1) larger numbers of cannabis consumers had a subsequent heart attack ( $67 \%$ vs. $41 \%$ of nonconsumers). This is also true with rates of hospitalizations for recurrent percutaneous coronary intervention and coronary artery bypass graft; (2) cannabis consumers were likely to be middleaged (median age 52 compared to median age 72 for nonconsumers); black Americans (34\% vs. $10 \%$ of nonconsumers); and male ( $77 \%$ vs. $62 \%$ of nonconsumers). Cannabis consumers had lower rates of high blood pressure, diabetes, and high cholesterol, although it is unclear why these risk factors were low; (3) there was a concerning increase in the trend of cannabis consumption among patients who had already had a heart attack or coronary revascularization procedure; (4) another alarming finding was that the frequency of recurrent heart attacks and cardiac interventions was higher among cannabis consumers, although they had fewer risk factors for heart disease and younger.

\section{Genetic Links}

As with many other drug/chemical compounds, response to cannabis use has been shown to have a genetic component, as evidenced by studies showing links to either a specific single-nucleotide polymorphism, or insertion/ deletion sequence that cause changes to number of different types of genes, including receptors, metabolic pathway enzymes, and activators/repressors of signaling pathways 
[57]. Some of these altered genes themselves have been related to changes in substance abuse or addiction [58] and potentially cannabis-induced schizophrenia [59]. In humans, cannabis consumption was also related to significantly lower sperm concentration [60]. Findings point to possible pre-conception paternal reproductive risks associated with cannabis consumption [50]. Diseases affecting specific endocannabinoid receptors are also known [61, $62]$. Thus, investigating the role of genetics on cannabis responses to various systems and identifying any potential markers that overlap other disease indications could be of benefit to understanding the future of cannabis as a medicine. Identification of certain types of predisposition to cannabis-induced responses, especially in situations where individuals may not be consuming it knowingly would be of great benefit to developing future medical treatments or diagnosis would be of significant benefit. Thus, these studies also provide a basis for understanding how CSHS, may affect different segment of the population.

\section{Effect of Cannabis on Appetite}

Although existing data suggest that cannabis use influences food intake, appetite, and metabolism, human data in this regard are limited [63]. Farokhnia et al. [63] investigated the effects of cannabis use on peripheral concentrations of appetitive and metabolic hormones in a sample of cannabis users. The study was performed in a randomized, crossover, double-blind, and placebo-controlled manner. Twenty participants underwent 4 sessions during which oral cannabis, smoked cannabis, vaporized cannabis, or placebo was administered. Active compounds contained about $50 \mathrm{mg}$ of THC. Repeated blood samples were recorded measuring endocrine markers, like total ghrelin (hunger hormone), acyl-ghrelin, leptin, glucagon-like peptide-1 (GLP-1; satiety hormone), and insulin. Results showed a significant drug main effect, as well as a significant drug $\times$ time-point interaction effect on insulin. The spike in blood insulin concentrations observed under the placebo condition (likely due to intake of brownie) was blunted by cannabis administration. A significant drug main effect and trend-level drug $\times$ timepoint interaction effect was also detected for GLP-1, suggesting that GLP-1 concentrations were lower under cannabis than the placebo condition. Finally, a significant drug main effect was found for total ghrelin, suggesting that total ghrelin concentrations during the oral cannabis session were higher than the smoked and vaporized cannabis sessions. The study concluded that cannabis ad- ministration modulated blood concentrations of some appetitive and metabolic hormones, mainly insulin, in cannabis users.

The mechanism of these phenomena may be explained by ghrelin which is an endogenous regulator of energy homeostasis synthesized by the stomach to stimulate appetite and positive energy balance. Similarly, the endocannabinoid system is part of internal machinery controlling food intake and energy expenditure [64]. Alen et al. [64] investigated brain ghrelin actions on food intake in rats with different metabolic status (negative or equilibrate energy balance). They also tested a sub-anxiogenic ultra-low dose of the $\mathrm{CB}_{1}$ antagonist SR141716A (rimonabant) and the peripheral-acting $\mathrm{CB}_{1}$ antagonist LH-21 on ghrelin orexigenic actions. They make the following observations: (1) central administration of ghrelin promotes food intake in free-feeding animals but not in $24 \mathrm{~h}$ food-deprived or chronically food-restricted animals; (2) an ultra-low dose of SR141716A completely counteracts the orexigenic actions of central ghrelin in free-feeding animals; (3) the peripheral-restricted $\mathrm{CB}_{1}$ antagonist LH-21 blocks ghrelin-induced hyperphagia in free-feeding animals. The study highlights the importance of the animal's metabolic status for the effectiveness of ghrelin in promoting feeding and suggests that the peripheral endocannabinoid system may interact with ghrelin signal in the control of food intake under equilibrate energy balance conditions. Since weight gain may lead to obesity which is central to metabolic syndrome (type II diabetes, hypercholesteremia, and hypertension), it is prudent to be vigilant about the societal use of recreational cannabis.

\section{Overview}

Cannabis is presently the most consumed illicit substance in the world. With the present trend of legalization of cannabis in the USA, healthcare providers will encounter more users consuming cannabis recreationally over a wide range of ages and health states. Early indications suggest that many compounds found in cannabis have potential therapeutic benefit, either alone or in conjunction with other cannabinoid or terpene compounds [65]. Additionally, recent research shows patients with both type 1 and type 2 diabetes had positive response to the use of certain types of cannabis $[66,67]$. Thus, cannabis can potentially be used in the treatment of at least some CVD. We should not put too much emphasis on the potential benefits of cannabis smoking among patients with diabe-
Dabiri/Kassab 
tes when these studies did not include/assess the risk of $\mathrm{MI}$ in a high-risk population.

With increasing evidence that cannabis may interact with systems involved in various aspects of hemostasis, additional research into the potential benefits of various cannabis chemovars and their potential differences with respect to treatment efficacy of many disease types is needed to fill the gap in our knowledge. Included in that is the need for additional research that addresses any potential hazards of the compounds themselves or methods of using cannabis that could potentially trigger a downstream reaction.

The justification for examining cannabis consumption in relation to cardiometabolic conditions is that cardiometabolic conditions are among the leading causes of mortality and are highly prevalent in the USA. A literature search indicates that relatively little is known about the relationship of cannabis use to CVD outcomes. There is clearly a great deal to learn about CV effects of cannabis. Understanding the mechanism through which cannabis causes infarction may provide insight into the triggering of MI. It is important to find a safe window of applications (i.e., dose limit) whereby positive medical aspect of the cannabis can be realized. Studies that specifically look at CV effects of cannabis when administered by nonsmoking routes may be interesting. For example, does cannabis administered without smoking affect heart rate, blood pressure, and thrombocyte aggregation measures? The effect of cannabis on CV should be extended to adolescence since there is dearth of data in the population.

It is likely that growing trend toward legalization of cannabis may encourage physicians and patients to talk more comfortably about the use which could allow greater data base and thus increase the insights into health effects. Research directions may include more studies of CVD outcomes and association of cannabis with risk factors, studies of metabolic and physiological effects of chronic cannabis consumption that may affect CVD risk, and increased understanding of the role of the cannabinoid receptor system in CV regulation since there are anecdotal evidence that there might not be significant $\mathrm{CV}$ adverse effects in medicinal use. Cooperation between the physicians and patients in terms of precise diagnosis of the $\mathrm{CV}$ and other conditions of the patient, and appropriate selection of cannabis could potentially eliminate the adverse effects of the product.

Research that identifies any potential effects of CSHS on not only respiratory and/or endothelial function, but also on potential changes in cognitive function, is crucial

Effects of Cannabis on the Cardiovascular System to understanding the potential effect of CSHS on the general populace, if consumption in public/general access areas is being considered. Any potential health effects of CSHS on the population should be investigated to provide policy makers the data upon which to make sound policy decisions when considering allowance of consumption of cannabis in public and exempting cannabis from smoke-free laws. The main goals should be to: (1) determine if CSHS caused any measurable changes in (a) respiratory function, (b) endothelial function, and (c) cognitive function; (2) determine the doses at which any potential changes occur; (3) determine the maximum acceptable dose for CSHS, if any, with respect to each functional category (e.g., $1[\mathrm{a}]$, [b], or [c]); (4) identify genetic markers associated with predisposition toward or against cardiac adverse reaction to the use of cannabis; and (5) determine if the levels detected in research goals $1(\mathrm{a}-\mathrm{c})$, 2 , and 3 , are different between the genetic groups identified in research goal mentioned above. These suggestions require longitudinal multivariable investigation to study the association of cannabis with CV events. The studies should be focused on individuals who do not smoke tobacco to isolate the effect of CSHS.

Spreading awareness regarding the potential risk of recurrent heart attacks in middle-aged, African-American, and male cannabis consumers and screening them at an earlier age for potential risk factors of future heart attacks should be encouraged among physicians. Clearly, further research is needed to look at the $\mathrm{CV}$ effects of synthetic cannabinoids to inform the regulatory measures that are necessary to curb the spread of synthetic cannabinoids and to keep the risk/benefit ratio of the medicinal use of cannabis and related cannabinoids as low as possible. In addition, the role of medicinal cannabis including benefits and potential risks with regards to $\mathrm{CV}$ management need to be validated in large blinded, randomized studies.

Existing data suggest that cannabis use influences food intake which could result in weight gain and eventually obesity. It is well-known that obesity is an independent risk factor for CVD and one of the main causes of the increased risk of diseases such as dyslipidemia, insulin resistance, hypertension, and atherosclerosis in both adults and children [68].

\section{Conclusions}

Early indications suggest that many compounds found in cannabis have potential therapeutic benefit either alone or in conjunction with other cannabinoid or 
terpene compounds which are largely unexplored. With increasing evidence that cannabis could interact with systems involved in various aspects of hemostasis, additional research into the potential benefits of various cannabis and their potential differences with respect to treatment efficacy of many disease types is needed to fill the gap in our knowledge. Increased awareness regarding the potential risk of recurrent heart attacks in middle-aged, African-American, and male cannabis consumers and screening them at an earlier age for potential risk factors of future heart attacks should be encouraged among physicians and public. The role of medicinal cannabis including benefits and potential adverse effects, such as weight gain with regards to $\mathrm{CV}$ management, need to be validated in large blinded, randomized experiments.

\section{Acknowledgments}

The authors would like to thank Drs. Keith March and Reggie Gaudino for their valuable comments on the review of the paper. Dr. Reggie Gaudino contributed the Genetic Links section of the paper.

\section{Conflict of Interest Statement}

The authors have no financial conflicts of interest to declare.

\section{Funding Sources}

This work is funded by 3DT Holdings, LLC.

\section{Author Contributions}

The authors equally contributed to the writing of the manuscript.

\section{References}

1 Available from: https://www.grandviewresearch.com/press-release/global-legal-marijuana-market.

2 Available from: https://news.gallup.com/ poll/267698/support-legal-marijuanasteady-past-year.aspx.

3 Available from: https://news.gallup.com/ poll/194195/adults-say-smoke-marijuana. aspx.

4 National Academy of Sciences, Engineering, and Medicine. The health effects of cannabis and cannabinoids: the current state of evidence and recommendations for research. Washington, DC: The National Academies Press; 2017.

5 Wikipedia, cannabis (drug), March 2021.

6 Meacham MC, Paul MJ, Ramo DE. Understanding emerging forms of cannabis use through an online cannabis community: an analysis of relative post volume and subjective highness ratings. Drug Alcohol Depend. 2018;188:364-9.

7 Mackie K. Cannabinoid receptors: where they are and what they do. J Neuroendocrinol. 2008;20 Suppl 1:10-4.

8 Mozaffarian D, Benjamin EJ, Go AS, Arnett DK, Blaha MJ, Cushman M, et al. American heart association statistics committee, and stroke statistics subcommittee. Heart disease and stroke statistics-2016 update: a report from the American heart association. Circulation. 2016;133(4):e38-360.

9 Kochanek KD, Murphy SL, Xu J, Tejada-Vera B. Deaths: final data for 2014. Natl Vital Stat Rep. 2016;65(4):1-122.

10 Benjamin EJ, Muntner P, Alonso A, Bittencourt MS, Callaway CW, Carson AP, et al.
Heart disease and stroke statistics-2019 update: a report from the American heart association. Circulation. 2019 Jan 31;139(10):e56-528.

11 Pertwee RG. Targeting the endocannabinoid system with cannabinoid receptor agonists: pharmacological strategies and therapeutic possibilities. Philos Trans R Soc Lond B Biol Sci. 2012;367(1607):3353-63.

12 FDA approves first drug comprised of an active ingredient derived from marijuana to treat rare, severe forms of epilepsy, for immediate release; 2018.

13 Stanley CP, Hind WH, O'Sullivan SE. Is the cardiovascular system a therapeutic target for cannabidiol? Br J Clin Pharmacol. 2013;75(2): $313-22$.

14 Jadoon KA, Tan GD, O'Sullivan SE. A single dose of cannabidiol reduces blood pressure in healthy volunteers in a randomized crossover study. JCI Insight. 2017;2(12):e93760.

15 Morgan CJ, Das RK, Joye A, Curran HV, Kamboj SK. Cannabidiol reduces cigarette consumption in tobacco smokers: preliminary findings. Addict Behav. 2013;38(9): 2433-6.

16 Hurd YL, Yoon M, Manini AF, Hernandez S, Olmedo R, Ostman M, et al. Early phase in the development of cannabidiol as a treatment for addiction: opioid relapse takes initial center stage. Neurotherapeutics. 2015;12(4):807-15.

17 Devinsky O, Cilio MR, Cross H, FernandezRuiz J, French J, Hill C, et al. Cannabidiol: pharmacology and potential therapeutic role in epilepsy and other neuropsychiatric disorders. Epilepsia. 2014;55(6):791-802.

18 Blessing EM, Steenkamp MM, Manzanares J, Marmar CR. Cannabidiol as a potential treat- ment for anxiety disorders. Neurotherapeutics. 2015;12(4):825-36.

19 Cheng D, Spiro AS, Jenner AM, Garner B, Karl T. Long-term cannabidiol treatment prevents the development of social recognition memory deficits in alzheimer's disease transgenic mice. J Alzheimers Dis. 2014;42(4): 1383-96.

20 Zuardi AW, Crippa JA, Hallak JE, Bhattacharyya S, Atakan Z, Martin-Santos R, et al. A critical review of the antipsychotic effects of cannabidiol: 30 years of a translational investigation. Curr Pharm Des. 2012; 18:5131-40.

21 Lehmann C, Fisher NB, Tugwell B, Szczesniak A, Kelly M, Zhou J. Experimental cannabidiol treatment reduces early pancreatic inflammation in type 1 diabetes. Clin Hemorheol Microcirc. 2016;64(4):655-62.

22 Massi P, Solinas M, Cinquina V, Parolaro D. Cannabidiol as potential anticancer drug. $\mathrm{Br} \mathrm{J}$ Clin Pharmacol. 2013;75(2):303-12.

23 Blasco-Benito S, Seijo-Vila M, Caro-Villalobos M, Tundidor I, Andradas C, GarcíaTaboada E, et al. Appraising the "entourage effect": antitumor action of a pure cannabinoid versus a botanical drug preparation in preclinical models of breast cancer. Biochem Pharmacol. 2018;157:285-93.

24 McLaren J, Swift W, Dillon P, Allsop S. Cannabis potency and contamination: a review of the literature. Addiction. 2008;103(7):11009.

25 Busse F, Omidi L, Timper K, Leichtle A, Windgassen $M$, Kluge E, et al. Lead poisoning due to adulterated Marijuana. N Engl J Med. 2008;358(15):1641-2. 
26 Randerson J. Warning issued over cannabis adulterated with glass beads; 2007. Available from: https: //www.theguardian.com/society/2007/jan/12/drugsandalcohol.drugs. 2007.

27 McPartland JM, Blanchon DJ, Musty RE. Cannabimimetic effects modulated by cholinergic compounds. Addict Biol. 2008;13(3): 411-5.

28 Thomas BF, Pollard GT. Preparation and distribution of cannabis and cannabis-derived dosage formulations for investigational and therapeutic use in the United States. Front Pharmacol. 2016;7:285.

29 Hermanns-Clausen M, Kithinji J, Spehl M, Angerer V, Franz F, Eyer F, et al. Adverse effects after the use of JWH-210: a case series from the EU Spice II plus project. Drug Test Anal. 2016;8(10):1030-8

30 Huestis MA. Human cannabinoid pharmacokinetics. Chem Biodivers. 2007;4:1770-804.

31 Tait RJ, Caldicott D, Mountain D, Hill SL, Lenton S. A systematic review of adverse events arising from the use of synthetic cannabinoids and their associated treatment. Clin Toxicol. 2016;54(1):1-13.

32 NIDA (National Institute on Drug Abuse) Drug facts: spice (synthetic marijuana). Rockville, MD: US Department of Health and $\mathrm{Hu}$ man Services, National Institutes of Health; 2012.

33 Atik SU, Dedeoğlu R, Varol F, Çam H, Eroğlu AG, Saltık L. Cardiovascular side effects related with use of synthetic cannabinoids "bonzai": two case reports. Turkish Pediatric Arsivi. 2015;50(1):61-4.

34 Pacher P, Steffens S, Haskó G, Schindler TH, Kunos G. Cardiovascular effects of marijuana and synthetic cannabinoids: the good, the bad, and the ugly. Nat Rev Cardiol. 2018; 15(3):151-66.

35 Sidney S. Cardiovascular consequences of Marijuana use. J Clin Pharmacol. 2002; 42(Suppl 1):64S-70S.

36 Sarafian TA, Magallanes JA, Shau H, Tashkin $\mathrm{D}$, Roth MD. Oxidative stress produced by marijuana smoke: an adverse effect enhanced by cannabinoids. Am J Respir Cell Mol Biol. 1999;20:1286-93.

37 Tashkin DP, Coulson AH, Clark VA, Simmons M, Bourque LB, Duann S, et al. Respiratory symptoms and lung function in habitual heavy smokers of marijuana alone, smokers of marijuana and tobacco, smokers of tobacco alone, and nonsmokers. Am Rev Respir Dis. 1987;135(1):209-16.

38 Bloom JW, Kaltenborn WT, Paoletti P, Camilli A, Lebowitz MD. Respiratory effects of non-tobacco cigarettes. Br Med J. 1987;295: $1516-8$.

39 Higgins M, Keller JB, Wagenknecht LE Townsend MC, Sparrow D, Jacobs DR Jr, et al. Pulmonary function, and cardiovascular risk factor relationships in black and in white young men and women: the CARDIA study. Chest. 1991;99:315-22.

40 Friedman GD, Klatsky AL, Siegelaub AB. The leukocyte count as a predictor of myocardial infarction. N Engl J Med. 1974;290(23):1275-

41 Iribarren C, Sidney S, Bild DE, Liu K, Markovitz JH, Roseman JM, et al. Association of hostility with coronary artery calcification in young adults: the CARDIA study. Coronary artery risk development in young adults. JAMA. 2000;283:2546-51.

42 Scherwitz LW, Perkins LL, Chesney MA, Hughes GH, Sidney S, Manolio TA. Hostility and health behaviors in young adults: the CARDIA study. Coronary artery risk development in young adults study. Am J Epidemiol. 1992;136:136-45.

43 Friedman GD, Tekawa I, Grimm RH, Manolio T, Shannon SG, Sidney S. The leucocyte count: correlates and relationship to coronary risk factors: the CARDIA study. Int J Epidemiol. 1990;19(4):889-93.

44 Mittleman MA, Lewis RA, Maclure M, Sherwood JB, Muller JE. Triggering myocardial infarction by Marijuana. Circulation. 2001; 103(23):2805-9.

45 Gleerup HB, Dahm CC, Thim T, Jensen SE, Jensen LO, Kristensen SD, et al. Smoking is the dominating modifiable risk factor in younger patients with STEMI. Eur Heart J Acute Cardiovasc Care. 2020;9(1):70-5.

46 Lindsay AC, Foale RA, Warren O, Henry JA. cannabis as a precipitant of cardiovascular emergencies. Int J Cardiol. 2005;104:230-2.

47 Aryana A, Williams MA. Marijuana as a trigger of cardiovascular events: speculation or scientific certainty? Int J Cardiol. 2007;118:141-4.

48 Gaziano JM. Marijuana use among those at risk for cardiovascular events. Am Heart J. 2008;155(3):395-6.

49 Mukamal KJ, Maclure M, Muller JE, Mittleman MA. An exploratory prospective study of Marijuana use and mortality following acute myocardial infarction. Am Heart J. 2008;155: 465-70.

50 Frost L, Mostofsky E, Rosenbloom JI, Mukamal KJ, Mittleman MA. Marijuana use and long-term mortality among survivors of acute myocardial infarction. Am Heart J. 2013; 165(2):170-5.

51 Jouanjus E, Lapeyre-Mestre M, Joelle M. cannabis use: signal of increasing risk of serious cardiovascular disorders. The French association of the regional abuse and dependence monitoring centers (CEIP-A) working group on cannabis complications. J Am Heart Assoc. 2014;3:e000638.

52 Franz CA, Frishman WH. Marijuana use and cardiovascular disease. Cardiol Rev. 2016; 24(4):158-62.

53 Marchetti D, Spagnolo A, De Matteis V, Filograna L, De Giovanni N. Coronary thrombosis and Marijuana smoking: a case report and narrative review of the literature. Drug Test Anal. 2016;8:56-62.

54 Rumalla K, Reddy AY, Mittal MK. Recreational Marijuana use and acute ischemic stroke: a population-based analysis of hospitalized patients in the United States. J Neurol Sci. 2016;364:191-6.
55 Wang X, Derakhshandeh R, Liu J, Narayan $S$, Nabavizadeh P, Le S, et al. One minute of Marijuana secondhand smoke exposure substantially impairs vascular endothelial function. J Am Heart Assoc. 2016;5: 003858.

56 Bhuva R. Marijuana use associated with complications after heart attack or procedures. Am Heart Assoc. 2020.

57 Agrawal A, Lynskey MT. Candidate genes for cannabis use disorders: findings, challenges and directions. Addiction. 2009;104(4):51832.

58 Herman AI, Kranzler HR, Cubells JF, Gelernter J, Covault J. Association study of the CNR1 gene exon 3 alternative promoter region polymorphisms and substance dependence. Am J Med Genet B Neuropsychiatr Genet. 2006; 141B(5):499-503.

59 Morgan CJ, Freeman TP, Powell J, Curran HV. AKT1 genotype moderates the acute psychotomimetic effects of naturalistically smoked cannabis in young cannabis smokers. Transl Psychiatry. 2016;6(6):e738.

60 Murphy SK, Itchon-Ramos N, Visco Z Huang Z, Grenier C, Schrott R, et al. Cannabinoid exposure and altered DNA methylation in rat and human sperm. Epigenetics. 2018;13(12):1208-21.

61 Glass M, Faull RL, Dragunow M. Loss of cannabinoid receptors in the substantia nigra in Huntington's disease. Neuroscience. 1993; 56(3):523-7.

62 Stempel AV, Stumpf A, Zhang HY, Özdoğan T, Pannasch U, Theis AK, et al. Cannabinoid type 2 receptors mediate a cell type-specific plasticity in the hippocampus. Neuron. 2016; 90:795-809.

63 Farokhnia M, McDiarmid GR, Newmeyer MN, Munjal V, Abulseoud OA, Huestis MA, et al. Effects of oral, smoked, and vaporized cannabis on endocrine pathways related to appetite and metabolism: a randomized, double-blind, placebo-controlled, human laboratory study. Transl Psychiatry. 2020;10: 71.

64 Alen F, Crespo I, Ramírez-López MT, Jagerovic N, Goya P, de Fonseca FR, et al. Ghrelin-induced orexigenic effect in rats depends on the metabolic status and is counteracted by peripheral CB1 receptor antagonism. PLoS One. 2013;8(4):e60918.

65 Russo EB. Taming THC: potential cannabis synergy and phytocannabinoid-terpenoid entourage effects. Br J Pharmacol. 2011;163: 1344-64.

66 Akturk HK, Taylor DD, Camsari UM, Rewers A, Kinney GL, Shah VN. Association between cannabis use and risk for diabetic ketoacidosis in adults with type 1 diabetes. JAMA Intern Med. 2019;179(1):115.

67 Muniyappa R, Sable S, Ouwerkerk R, Mari A, Gharib AM, Walter M, et al. Metabolic effects of chronic cannabis smoking. Diabetes Care. 2013;36(8):2415-22.

68 Cercato C, Fonseca FA. Cardiovascular risk and obesity. Diabetol Metab Syndr. 2019;11: 74. 